\title{
Ursolic acid promotes colorectal cancer cell apoptosis and inhibits cell proliferation via modulation of multiple signaling pathways
}

\author{
JIUMAO LIN ${ }^{1,2^{*}}$, YOUQIN CHEN ${ }^{3 *}$, LIHUI WEI $^{1,2}$, ALING SHEN $^{1,2}$, \\ THOMAS J. SFERRA ${ }^{3}$, ZHENFENG HONG $^{1}$ and JUN PENG ${ }^{1,2}$
}

\author{
${ }^{1}$ Academy of Integrative Medicine and ${ }^{2}$ Fujian Key Laboratory of Integrative Medicine on Geriatric, \\ Fujian University of Traditional Chinese Medicine, Fuzhou, Fujian 350122, P.R. China; ${ }^{3}$ Case Western Reserve \\ University School of Medicine, Rainbow Babies and Children's Hospital, Cleveland, OH 44106, USA
}

Received June 5, 2013; Accepted July 17, 2013

DOI: $10.3892 /$ ijo.2013.2040

\begin{abstract}
The development of colorectal cancer (CRC) is strongly correlated with the aberrant activation of multiple intracellular signaling transduction cascades including STAT3, ERK, JNK and p38 pathways which usually function redundantly. In addition, crosstalk between these pathways forms a complicated signaling network that is regulated by compensatory mechanisms. Therefore, most of the currently used and single-target-based antitumor agents might not always be therapeutically effective. Moreover, long-term use of these agents often generates drug resistance. These problems highlight the urgent need for the development of novel anticancer chemotherapies. Ursolic acid (UA) is a major active compound present in many medicinal herbs that have long been used for the clinical treatment of CRC. Although previous studies have demonstrated an antitumor effect for UA, the precise mechanisms of its tumoricidal activity are not well understood. In the present study, using CRC mouse xenograft model and the HT-29 human colon carcinoma cell line, we evaluated the efficacy of UA against tumor growth in vivo and in vitro and investigated the underlying molecular mechanisms. We found
\end{abstract}

Correspondence to: Dr Jun Peng, Academy of Integrative Medicine Biomedical Research Center, 1 Huatuo Road, Minhou Shangjie, Fuzhou, Fujian 350122, P.R. China

E-mail: pjunlab@hotmail.com

*Contributed equally

Abbreviations: UA, ursolic acid; CRC, colorectal cancer; DMSO, dimethyl sulfoxide; MTT, 3-(4,5-dimethyl-thiazol-2-yl)2,5-diphenyltetrazolium bromide; IHS, immunohistochemical staining; TUNEL, terminal deoxynucleotidyl transferase-mediated dUTP nick end-labeling; PCNA, proliferating cell nuclear antigen; STAT3, signal transducer and activator of transcription 3; Erk1/2, extracellular signal-regulated protein kinases 1 and 2; JNK, c-Jun $\mathrm{N}$-terminal kinase; p38, p38 mitogen-activated protein kinase

Key words: ursoloic acid, colorectal cancer, signaling pathway, multi-target-based agent that UA inhibits cancer growth without apparent toxicity. Furthermore, UA significantly suppresses the activation of several CRC-related signaling pathways and alters the expression of critical target genes. These molecular effects lead to the induction of apoptosis and inhibition of cellular proliferation. These data demonstrate that UA possesses a broad range of anticancer activities due to its ability to affect multiple intracellular targets, suggesting that UA could be a novel multipotent therapeutic agent for cancer treatment.

\section{Introduction}

Colorectal carcinoma (CRC) is a serious global health problem with over one million new cases and half a million deaths worldwide each year (1). To date, chemotherapy is the main therapeutic approach for invasive and metastatic CRC. Despite recent advances in this area, 5-fluorouracil (5-FU)-based regimens continue to be the standard therapeutic approach for patients with advanced CRC (2). However, due to drug resistance and an unacceptable level of toxicity, 5-FU-based regimens produce objective response rates of $<40 \%(3,4)$.

The pathogenic mechanisms underlying CRC development are complex and heterogeneous and involve multiple cellular signaling transduction pathways including signal transducer and activator of transcription 3 (STAT3), extracellular regulated protein kinases (ERK), c-Jun $\mathrm{NH}(2)$-terminal protein kinases (JNK) and p38 kinase. STAT3 is a transcription factor that plays an essential role in cell survival and proliferation (5). After activation via phosphorylation by receptor and non-receptor protein tyrosine kinases, STAT3 proteins in the cytoplasm dimerize and translocate to the nucleus where they regulate the expression of critical genes involved in cell proliferation and survival $(5,6)$. Constitutive activation of STAT3 is highly correlated with the development of numerous types of cancer including CRC and commonly suggests a poor prognosis (7-10). MAPK signaling is one of the major cell-survival and proliferation pathways. In mammals, there are three major subfamilies of MAPK, including ERK, JNK and $\mathrm{p} 38$. Activation of all MAPK pathways is regulated by a central three-tiered kinase core consisting of MAPK kinase kinase (MAP3K), MAPK kinase (MAP2K) and MAPK; wherein MAP3K phosphorylates MAP2K and activated 
MAP2K in turn phosphorylates and activates MAPK $(11,12)$. MAPK signaling is important in intestinal epithelial differentiation (13); however, aberrant activation of MAPK pathway is involved in colon carcinogenesis (14-17).

The molecular pathways described above modulate the expression of key genes involved in the regulation of cell proliferation, apoptosis and angiogenesis and are participants in the processes of induction, progression and metastasis of colorectal carcinoma. Thus, each serves as a potential target for novel chemotherapeutics. It is noteworthy that these signaling pathways usually have functional redundancy. In addition, there is crosstalk between these pathways, forming a complicated and robust cellular signal transduction network that is regulated by compensatory mechanisms. Therefore, inhibitors that target only a single pathway might not be effective initially and their long-term use might generate drug resistance (18). These problems highlight the need for the identification of novel cancer chemotherapies. Natural products, such as traditional Chinese medicines (TCMs), have been used clinically to treat various types of diseases including cancer (19). Their effect, in general, is believed to be the result of targeting multiple molecular pathways. Moreover, TCMs have relatively fewer side effects as compared to modern chemotherapeutics. Therefore, TCMs have gained recent attention as potential chemotherapeutics for CRC.

Many traditional Chinese medicinal herbs, such as Hedyotic diffusa, Spica prunella and Scutellaria barbata, have long been used in China for the clinical treatment of CRC (20-23). Ursolic acid (UA), a pentacyclic triterpene acid, is a biologically active compound present in these herbs. Previous studies report that UA exhibits a broad range of pharmacological properties such as anti-inflammatory, antiviral, antioxidant, hepatoprotective, cytotoxic, antitumor, anti-angiogenesis and anti-metastatic activities (24). Recent studies have shown that UA inhibits the proliferation and induces apoptosis and/inhibits proliferation of colon carcinoma cells $(25,26)$. In this study, we further evaluated the efficacy of UA as a therapeutic agent for CRC in vivo and in vitro and investigated the underlying molecular mechanisms of its action.

\section{Materials and methods}

Materials and reagents. Ursolic acid (UA) was purchased from Sigma-Aldrich Chemical (St. Louis, MO, USA). Matrigel was provided by Becton-Dickinson (San Jose, CA, USA). Dulbecco's modified Eagle's medium (DMEM), fetal bovine serum (FBS), penicillin-streptomycin, trypsin-EDTA and TRIzol reagent were purchased from Invitrogen (Carlsbad, CA, USA). TUNEL assay kit (TumorTACS in situ) was purchased from R\&D Systems (Minneapolis, MN, USA). BCA Protein Assay kit was purchased from Tiangen Biotech Co., Ltd. (Beijing, China). All antibodies were purchased from Cell Signaling Technology (CST, Beverly, MA, USA). Bio-Plex phosphoprotein assay kits were purchased from Bio-Rad (Hercules, CA, USA). All other chemicals, unless otherwise stated, were obtained from Sigma-Aldrich.

Cell culture. Human colon carcinoma HT-29 cells were purchased from American Type Culture Collection (ATCC, Manassas, VA, USA). HT-29 cells were grown in DMEM.
DMEM was supplemented with $10 \%$ (v/v) FBS, $100 \mathrm{U} / \mathrm{ml}$ penicillin and $100 \mu \mathrm{g} / \mathrm{ml}$ streptomycin. The cells were cultured at $37^{\circ} \mathrm{C}, 5 \% \mathrm{CO}_{2}$ and in a humidified environment.

Animals. Male BALB/c athymic (nude) mice (with an initial body weight of 20-22 g) were obtained from Shanghai SLAC Laboratory Animal Co., Ltd. (Shanghai, China) and housed under pathogen-free conditions with controlled temperature $\left(22^{\circ} \mathrm{C}\right)$, humidity and a 12-h light/dark cycle. Food and water were given ad libitum throughout the experiment. All animal treatments were performed strictly in accordance with international ethical guidelines and the National Institutes of Health Guide concerning the Care and Use of Laboratory Animals. The experiments were approved by the Institutional Animal Care and Use Committee of Fujian University of Traditional Chinese Medicine.

In vivo nude mice xenograft study. CRC xenograft mice were produced with HT-29 cells as described previously (21). At 5 days following xenograft implantation (tumor size $\sim 3 \mathrm{~mm}$ in diameter), mice were randomized into two groups $(\mathrm{n}=10)$ and treated with UA (dissolved in PBS) $12.5 \mathrm{mg} / \mathrm{kg}$ or saline by daily intraperitoneal injection, 6 days a week for 16 days. Body weight and tumor size were measured. Tumor size was determined by measuring the major (L) and minor (W) diameter with a caliper. The tumor volume was calculated according to the following formula: tumor volume $=\pi / 6 \mathrm{xLxW}^{2}$. At the end of the experiment, the animals were anaesthetized with pelltobarbitalum natricum and tumors were excised and weighed.

Cell viability evaluation by MTT assay. UA was dissolved in DMSO and diluted to working concentrations with culture medium. The final concentration of DMSO in the medium for all cell-based experiments was $0.1 \%$. HT- 29 cells were seeded into 96-well plates at a density of $1.0 \times 10^{4}$ cells/well in $0.1 \mathrm{ml}$ medium. The cells were treated with various concentrations of UA for $24 \mathrm{~h}$ or with $40 \mu \mathrm{M}$ of UA for different periods of time. Treatment with $0.1 \%$ DMSO was included as the vehicle control. At the end of the treatment, $10 \mu 1$ MTT [ $5 \mathrm{mg} / \mathrm{ml}$ in phosphate-buffered saline (PBS)] were added to each well and the samples were incubated for an additional $4 \mathrm{~h}$ at $37^{\circ} \mathrm{C}$. The purple-blue MTT formazan precipitate was dissolved in $100 \mu 1$ DMSO. Absorbance was measured at $570 \mathrm{~nm}$ using an ELISA reader (BioTek, Model EXL800, USA).

Colony formation assay. HT-29 cells were seeded into 6-well plates at a density of $2 \times 10^{5}$ cells/well and treated with various concentrations of UA for $24 \mathrm{~h}$. The cells were harvested, resuspended in medium in the absence of UA and reseeded into 6 -well plates at a density of $1.5 \times 10^{3}$ cells/well. After incubation for 7 days in a $37^{\circ} \mathrm{C}$ humidified incubator with $5 \% \mathrm{CO}_{2}$, colonies were counted under light microscopy. Cell survival was calculated by normalization to the survival of control (untreated) cells.

Cell cycle analysis. Cell cycle analysis was carried out by flow cytometry using FACS analysis with propidium iodide (PI) staining. HT-29 cells were treated with various concentrations of UA for $24 \mathrm{~h}$, harvested, adjusted to a concentration 
of $1 \times 10^{6}$ cells $/ \mathrm{ml}$ and fixed in $70 \%$ ethanol at $4^{\circ} \mathrm{C}$ overnight The fixed cells were washed twice with cold PBS and incubated for $30 \mathrm{~min}$ with RNase $(8 \mu \mathrm{g} / \mathrm{ml})$ and PI $(10 \mu \mathrm{g} / \mathrm{ml})$. The fluorescent signal was detected through the FL2 channel and the proportion of DNA in different phases was analyzed using ModfitLT Version 3.0 (Verity Software House, Topsham).

Apoptosis detection in CRC tumor tissues by TUNEL staining. Formalin-fixed (12 h) and paraffin-embedded sections of tumors (4- $\mu$ m-thick) were analyzed by TUNEL staining using TumorTACS in situ kit (R\&D Systems). Apoptotic cells were counted as DAB-positive cells (brown stained) at five arbitrarily selected microscopic fields at a magnification of $\mathrm{x} 400$. TUNEL-positive cells were counted as a percentage of the total cells.

Apoptosis detection in HT-29 cells by flow cytometry analysis with Annexin V/PI staining. After incubation with various concentrations of UA, apoptosis of HT-29 cells were determined by flow cytometry using a fluorescenceactivated cell sorting (FACS) caliber (Becton-Dickinson) and Annexin V-fluorescein isothiocyanate (FITC)/propidium iodide (PI) kit (Becton-Dickinson). Staining was performed according to the manufacturer's instructions. The percentage of cells in early apoptosis was calculated by Annexin V-positivity and PI-negativity and the percentage of cells in late apoptosis was calculated by Annexin V-positivity and PI-positivity.

DNA fragmentation analysis in HT-29 cells by gel electrophoresis. DNA fragmentation was determined using a DNA ladder detection kit (Invitrogen), following the manufacturer's instructions. Briefly, HT-29 cells were detached from the culture plates by scraping and washed in PBS. DNA was isolated from $2 \times 10^{6}$ cells. The DNA was analyzed after separation by gel electrophoresis. The DNA bands were examined using a gel documentation system (Model Gel Doc 2000, Bio-Rad).

Immunohistochemistical analysis of CRC tumor tissues. Six tumors were randomly selected from UA-treatment or control groups. Tumor tissues were fixed in $10 \%$ formaldehyde for $12 \mathrm{~h}$, paraffin-embedded, sectioned and placed on slides. The immunohistochemistical analysis was carried out as described previously (21). Briefly, the slides were subjected to antigen retrieval and endogenous peroxidase activity was quenched with hydrogen peroxide. Non-specific binding was blocked with normal serum in PBS (0.1\% Tween-20). Rabbit polyclonal antibodies against PCNA (in 1:200 dilution, CST) was used to detect the proteins. After staining, five high-power fields (at magnification of $\mathrm{x} 400$ ) were randomly selected in each slide. The proportion of positive cells in each field was determined using the true color multi-functional cell image analysis management system (Image-Pro Plus, Media Cybernetics, USA). To control for non-specific staining, PBS was used to replace the primary antibody as a negative control.

RT-PCR analysis. Total RNA was isolated from tumor tissues (three tumors were randomly selected from UA-treatment or control groups) or HT-29 cells with TRIzol reagent. Oligo(dT)primed RNA (1 $\mu \mathrm{g}$, isolated from tumor tissues or cells) was reverse-transcribed with SuperScript II reverse transcriptase (Promega, Madison, WI, USA) according to the manufacturer's instructions. The obtained cDNA was used to determine the level of Bcl-2, Bax, Cyclin D1, CDK4 and p21 mRNA by PCR with Taq DNA polymerase (Fermentas). GAPDH was used as an internal control. Samples were analyzed by gel electrophoresis (1.5\% agarose). The DNA bands were examined using a gel documentation system (Model Gel Doc 2000, Bio-Rad).

Western blot analysis. The western blot analysis was carried out as described previously (21). Briefly, tumors and HT-29 cells were separately lysed in mammalian cell lysis buffer with different protein inhibitor. Equal amounts of protein from each tumor or cell lysate was subjected to SDS-PAGE and transferred onto PVDF membranes. The membranes were blocked for $2 \mathrm{~h}$ with $5 \%$ non-fat dry milk and incubated with the desired primary antibody directed against p-STAT3, total-STAT3, Bcl-2, Bax, Cyclin D1, CDK4, p21, or $\beta$-actin (all in 1:1,000 dilutions) overnight at $4^{\circ} \mathrm{C}$. Appropriate HRP-conjugated secondary antibodies with chemiluminescence detection were used to image the antibody-detected proteins.

Bio-Plex phosphoprotein assay. Eight tumors were randomly selected from UA-treatment or control groups and homogenized. HT-29 cells $\left(2.5 \times 10^{5}\right)$ were seeded into $25 \mathrm{~cm}^{2}$ flasks in $5 \mathrm{ml}$ medium and treated with $40 \mu \mathrm{M}$ of UA for $24 \mathrm{~h}$. Tumor tissues and treated cells were lysed using a commercially available lysis kit (Bio-Rad Laboratories) and centrifuged at $14,000 \times \mathrm{g}$ for $15 \mathrm{~min}$. The protein extracts were quantified by BCA protein assay. The presence of p-ERK $1 / 2$, p-JNK and p-p38 was detected using a bead-based multiplex assay for phosphoproteins (Bio-Plex Phosphoprotein assay, Bio-Rad Laboratories) according to the manufacturer's protocol. Data was collected and analyzed using the Bio-Plex 200 suspension array system (Bio-Rad).

Statistical analysis. Data were presented as mean \pm SD for the indicated number of independently performed experiments. The data were analyzed using the SPSS package for Windows (Version 17.0). Statistical analysis was carried out with Student's t-test and ANOVA. Differences with $\mathrm{P}<0.05$ were considered to be statistically significant.

\section{Results}

UA inhibits tumor growth in colorectal cancer (CRC) xenograft mice. The in vivo antitumor effect of UA was evaluated through comparison of tumor weight and volume in treated and control CRC xenograft mice, while its adverse effects were determined in the same mice by measuring changes in body weight. As shown in Fig. 1A, UA treatment resulted in $55.9 \%$ less tumor volume as compared to control (control, $0.93 \pm 0.21$ $\mathrm{cm}^{3}$; UA-treatment, $\left.0.41 \pm 0.13 \mathrm{~cm}^{3}, \mathrm{P}<0.01\right)$. In a consistent manner, the tumor weights in the UA-treatment group was $65.7 \%$ less than in the control group (control, $0.67 \pm 0.15 \mathrm{~g}$; UA-treatment, $0.23 \pm 0.11 \mathrm{~g}, \mathrm{P}<0.01$, Fig. 1B). However, administration of UA had no effect on gain in body weight during the course of the study (Fig. 1C), suggesting that UA is potent in suppressing colorectal tumor growth in vivo, without noticeable toxicity. 

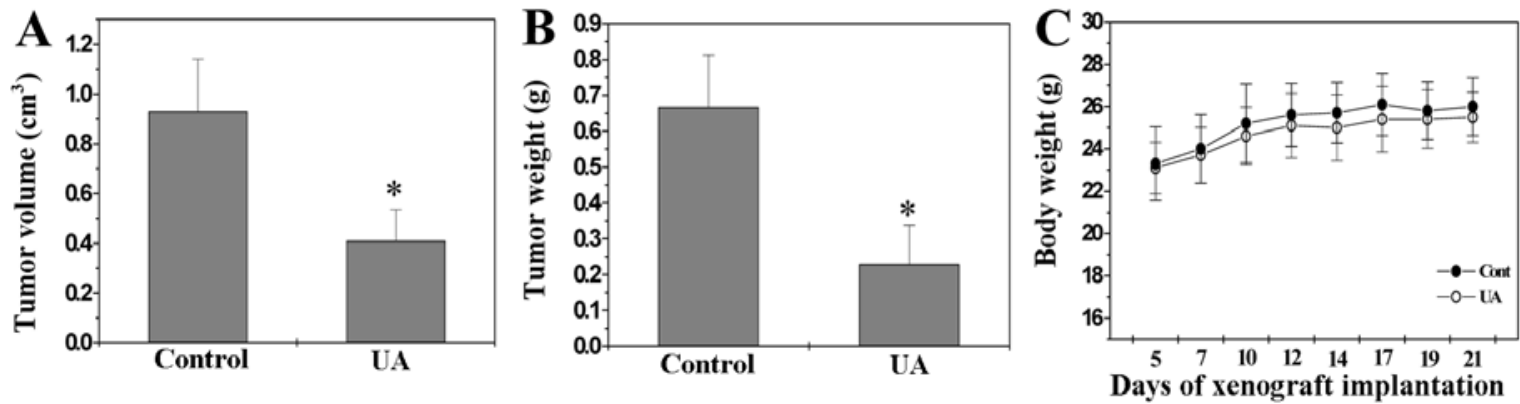

Figure 1. UA inhibits tumor growth in CRC xenograft mice. Tumor volume (A), tumor weight (B) and body weight C) were measured. Data shown are averages with $\mathrm{SD}$ (error bars) from 10 mice in each group. ${ }^{*} \mathrm{P}<0.01$, versus controls.
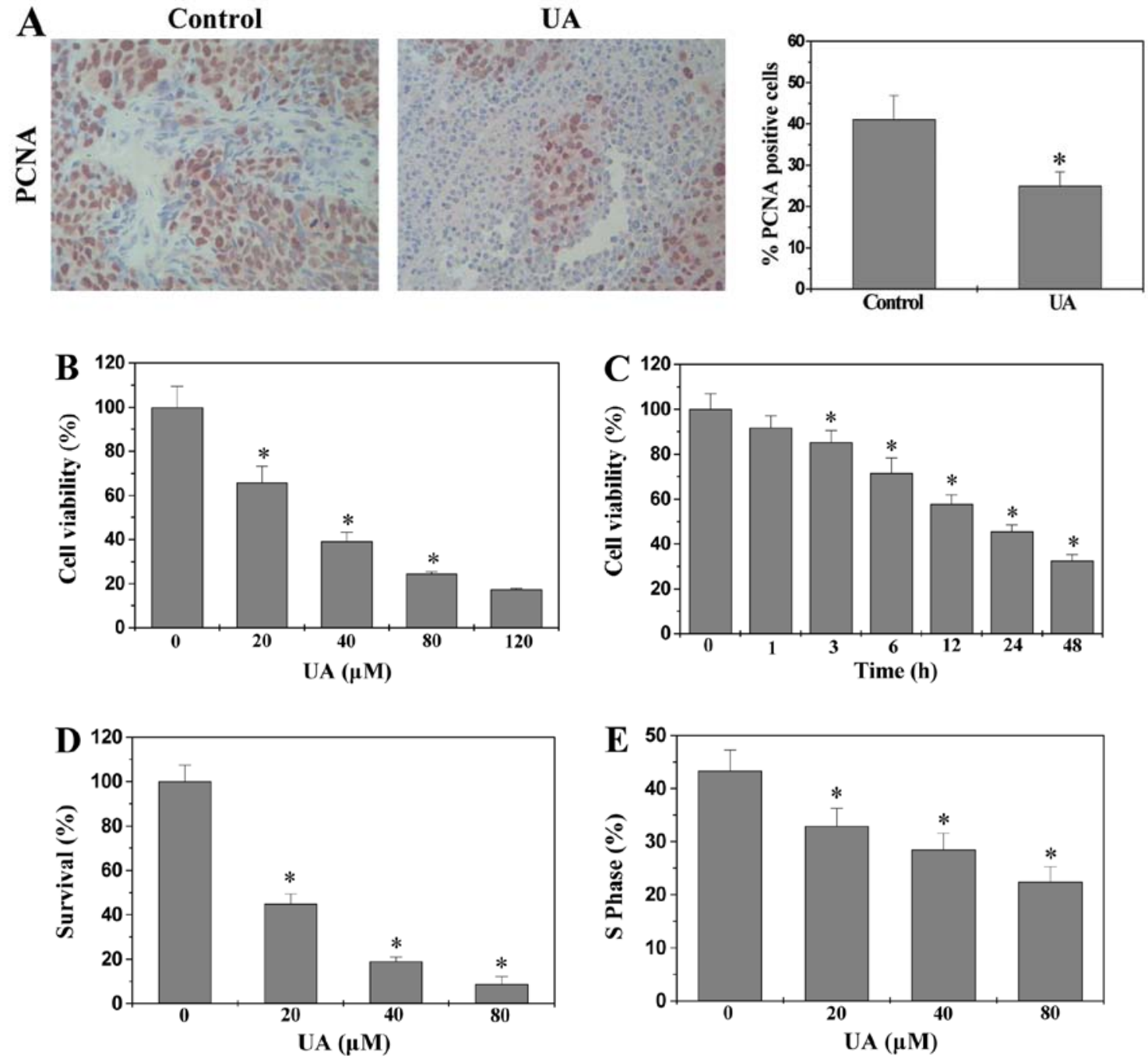

Figure 2. Effect of UA on cell proliferation in CRC xenograft mice and HT-29 cells. (A) PCNA assay in tumor tissues (x400). Data shown are averages with $\mathrm{SD}$ (error bars) from 6 individual mouse in each group. " $\mathrm{P}<0.01$, versus controls. (B and C) HT-29 cell viability was determined by the MTT assay after cells were treated with the indicated concentrations of UA for $24 \mathrm{~h}$ or with $40 \mu \mathrm{M}$ of UA for the indicated time periods. (D) HT-29 cell survival was evaluated by colony formation assay. The data were normalized to the viability or survival of control cells (100\%, treated with $0.1 \%$ DMSO vehicle). (E) HT-29 cell cycle was analyzed by FACS and the proportion of cells in S-phase was calculated. Data from (B-E) are averages with SD (error bars) from at least three independent experiments. ${ }^{*} \mathrm{P}<0.01$ versus controls.

UA inhibits proliferation of colorectal cancer cells via G1/S cell cycle arrest and modulation of Cyclin D1, CDK4 and p21 expression. The in vivo effect of UA on CRC cell prolif- eration was determined via immunohistochemical staining (IHS) for PCNA, a proliferation marker that is specifically expressed in proliferating cell nuclei. As shown in Fig. 2A, 
A

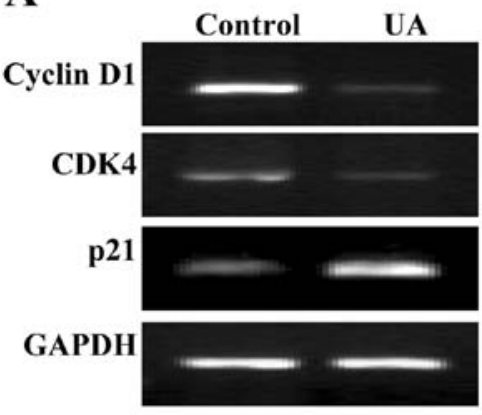

C

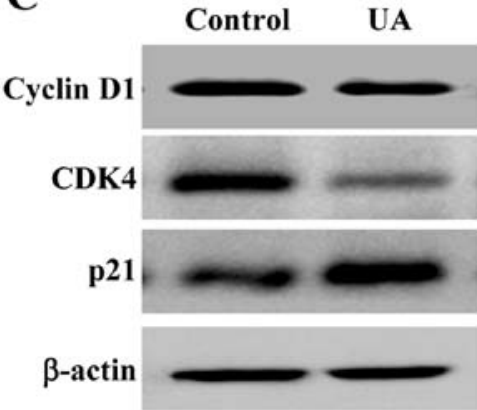

B

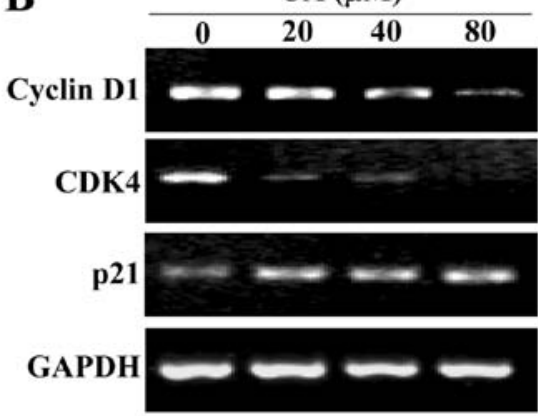

D

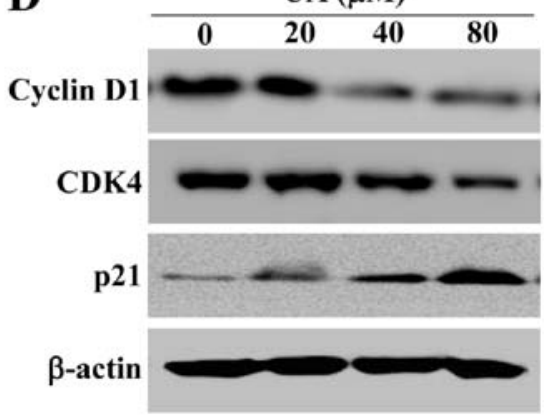

Figure 3. Effect of UA on the expression of Cyclin D1, CDK4 and p21 in CRC xenograft mice and HT-29 cells. The mRNA levels of Cyclin D1, CDK4 and p21 in tumor tissues (A) or HT-29 cells (B) were determined by RT-PCR. The protein expression levels of Cyclin D1, CDK4 and p21 in tumor tissues (C) or HT-29 cells (D) were determined by western blotting. GAPDH and $\beta$-actin were used as the internal controls for the RT-PCR or western blotting, respectively. Images are representatives of 3 mice in each group or of three independent cell-based experiments.

the percentage of PCNA-positive cells in tumor tissues from control and UA-treated CRC xenograft mice was $41.0 \pm 5.9 \%$ and $25.0 \pm 3.4 \%$, respectively $(\mathrm{P}<0.01)$, suggesting that UA represses cell proliferation in tumor tissues.

We next sought to examine the effect of UA on CRC cell proliferation in vitro. We treated human colon carcinoma HT-29 cells with various concentrations of UA and measured the amount of surviving cells with the MTT assay. As shown in Fig. 2B, treatment with $20-120 \mu \mathrm{M}$ of UA for $24 \mathrm{~h}$ dosedependently reduced the viability of HT-29 cells $(\mathrm{P}<0.01)$. The estimated half maximal inhibitory concentration $\left(\mathrm{IC}_{50}\right)$ of UA in HT-29 cells was $\sim 35 \mu \mathrm{M}$. We next evaluated the effect of UA on cell viability as a function of time. We found that treatment with UA $\left(40 \mu \mathrm{M}\right.$, approximately the $\left.\mathrm{IC}_{50}\right)$ led to a gradual decrease in cell viability with an increase in exposure time $(\mathrm{P}<0.01$, Fig. $2 \mathrm{C})$. We further verified these results using a colony formation assay. UA treatment dosedependently reduced the cell survival rate ( $\mathrm{P}<0.01$, Fig. 2D). Thus, UA inhibits CRC cell proliferation in vitro dose- and time-dependently.

G1/S transition is one of the two main cell cycle checkpoints that are critical for regulation of cell proliferation, which is strongly regulated by Cyclin D1, CDK4 and p21. We therefore investigated the effect of UA on the G1 to S progression and on the expression of these regulatory genes. Using PI staining followed by FACS analysis we found that the percentage HT-29 cells in S-phase following treatment with UA was decreased in a dose-dependent manner (Fig. 2E, $\mathrm{P}<0.01$ ). In addition, data from RT-PCR and western blot analysis showed that UA treatment significantly reduced the mRNA and protein levels of pro-proliferative Cyclin D1 and CKD4 both in CRC tumors and HT-29 cells, whereas the mRNA and protein levels of anti-proliferative p21 was significantly increased after UA treatment (Fig. 3, $\mathrm{P}<0.01$ ). These data together suggest that UA inhibits CRC cell proliferation through blockade of G1-S progression and the modulation of the expression of cell cycleregulatory genes.

UA induces colorectal cancer cell apoptosis via alteration in the Bax/Bcl-2 ratio. To further evaluate the effect of UA on CRC cell growth, we examined whether UA induces apoptosis. Apoptosis in tumors was evaluated via TUNEL. As shown in Fig. 4A, the percentage of TUNEL-positive cells was greater in tumors from UA-treated mice as compared to controls

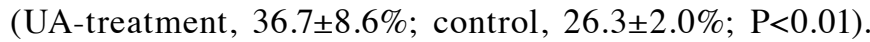
UA-induced apoptosis in vitro was examined using HT-29 cells and Annexin V/PI staining followed by FACS analysis. As shown in Fig. 4B and C, UA treatment increased the percent of cells undergoing apoptosis [early apoptosis found within the lower right (LR) and late apoptosis upper right (UR) quadrants in the FACS diagram] in a dose-dependent fashion $(\mathrm{P}<0.05)$. The in vitro pro-apoptotic activity of UA was confirmed by its effect on cellular DNA fragmentation, a typical feature of apoptosis. As shown in Fig. 4D, DNA extracted from HT-29 cells treated with UA displayed a characteristic ladder pattern of discontinuous DNA fragments.

To investigate the mechanism of the pro-apoptotic activity of UA, we examined its effect on the expression of Bcl-2 family proteins that are important regulators of apoptosis. As shown in Fig. 5A and B, UA significantly reduced anti-apoptotic 
A

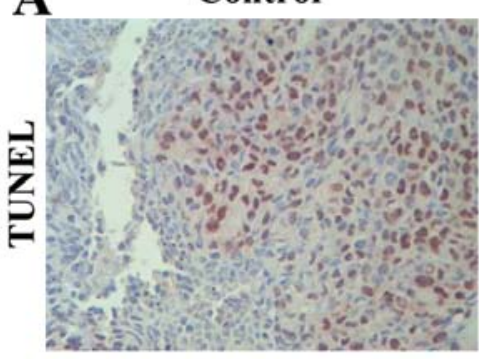

B
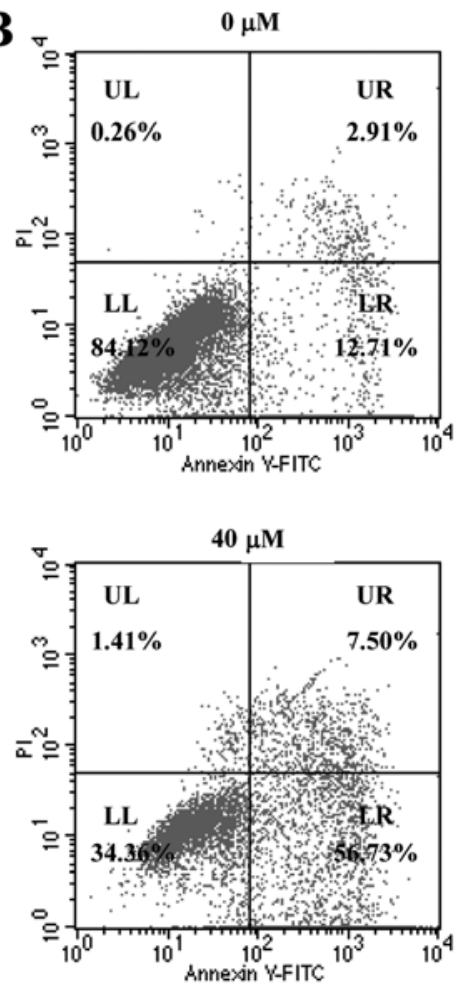

UA
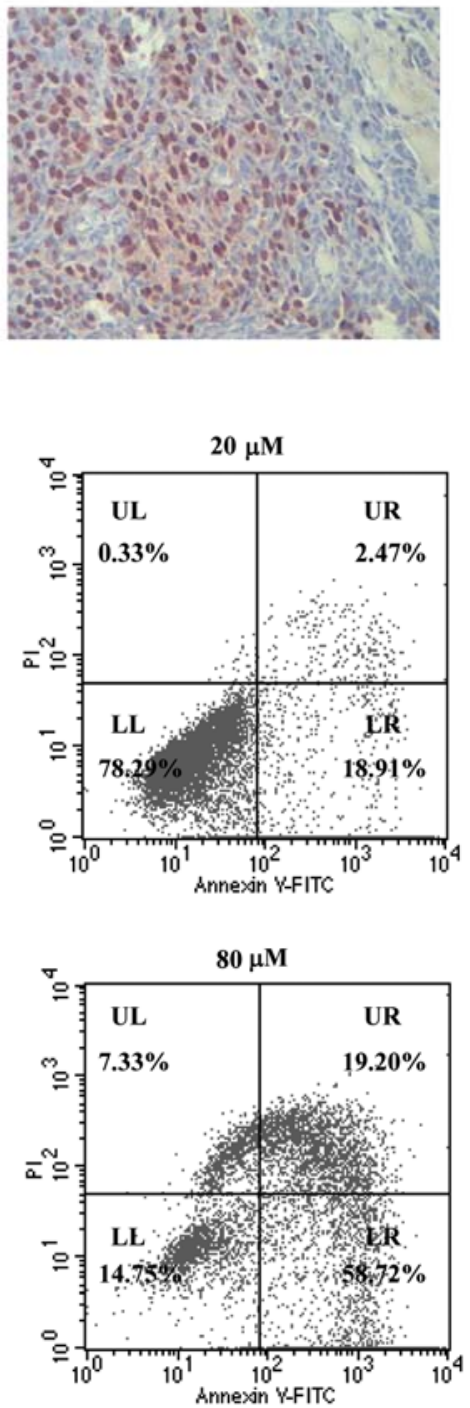
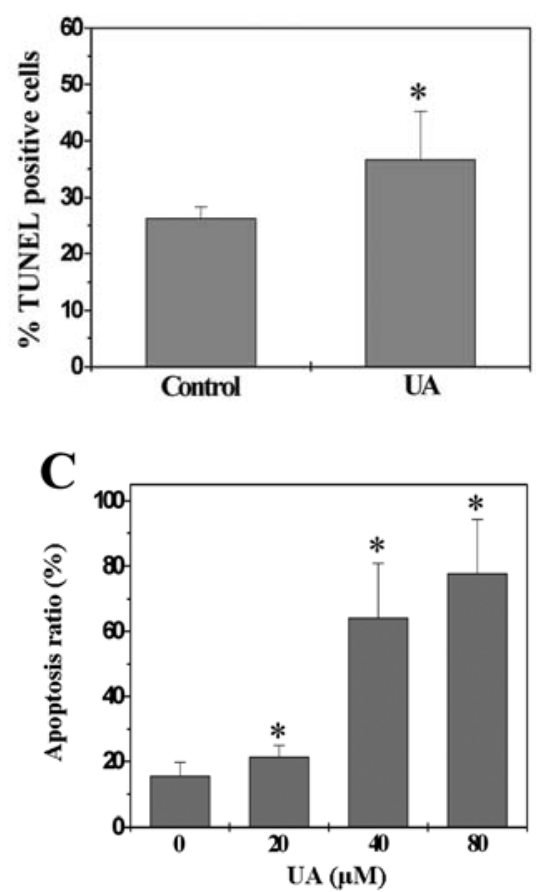

D

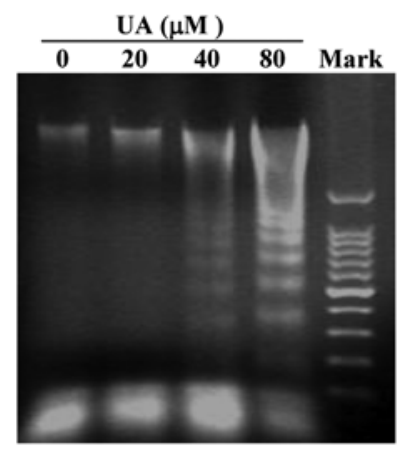

Figure 4. Effect of UA on cell apoptosis in CRC xenograft mice and HT-29 cells. (A) TUNEL assay in tumor tissues (x400). Data shown are averages with SD (error bars) from 6 mice in each group. ${ }^{*} \mathrm{P}<0.01$, versus controls. (B) Apoptosis of HT-29 cells were analyzed by FACS. Images are representative of three independent experiments. (C) Quantification of FACS analysis. The data shown are averages with SD (error bars) from three independent experiments. ${ }^{*}<0.01$, versus controls. (D) UA-induced DNA fragmentation in HT-29 cells. Images are representative of three independent experiments.

Bcl-2 mRNA level both in the tumors of CRC mice and HT-29 cells, whereas the level of pro-apoptotic Bax mRNA was significantly increased after UA treatment. The protein expression patterns of Bcl-2 and Bax were similar to the patterns observed for the respective mRNA (Fig. 5C and D). Taken together, these data demonstrate that UA promotes colorectal cancer cell apoptosis both in vivo and in vitro through an increase in the pro-apoptotic $\mathrm{Bax} / \mathrm{Bcl}-2$ ratio.

UA suppresses multiple signaling pathways in CRC cells. To further explore the underlying mechanisms of the observed anticancer activities of UA, we determined its effect on the activation of several CRC-related signal transduction cascades including STAT3, ERK, JNK and p38. Activation of STAT3 is mediated by its phosphorylation at tyrosine 705 , we therefore investigated the effect of UA on STAT3 activation in CRC by western blot analysis using an antibody that recognizes STAT3 phosphorylation at $\mathrm{Tyr}^{705}$. Since many cultured human cancer cell lines including HT-29 do not express constitutively phosphorylated STAT3 in vitro, we stimulated STAT3 activation in HT-29 cells with $10 \mathrm{ng} / \mathrm{ml}$ of IL-6 for $15 \mathrm{~min}$. As shown in Fig. 6A, UA profoundly inhibited IL-6 induced phosphorylation of STAT3 in HT-29 cells. In a consistent fashion, UA treatment significantly decreased the level of phosphorylated STAT3 in tumors of CRC xenograft mice (Fig. 6B). The levels of non-phosphorylated STAT3 in tumor HT-29 cells and tumor tissues remained unchanged with UA treatment.

The activation (phosphoralytion) of ERK1/2, JNK and p38 in CRC xenograft tumor tissues and HT-29 cells was determined by Bio-Plex Phospho-protein assay. As shown in Fig. 6C and D, after UA treatment the phosphorylation levels of ERK1/2, JNK and p38 in both tumors and HT-29 cells were decreased as compared to controls $(\mathrm{P}<0.01)$. Collectively, these data suggest that UA significantly suppresses the activation of multiple CRC-related signaling pathways. 
A

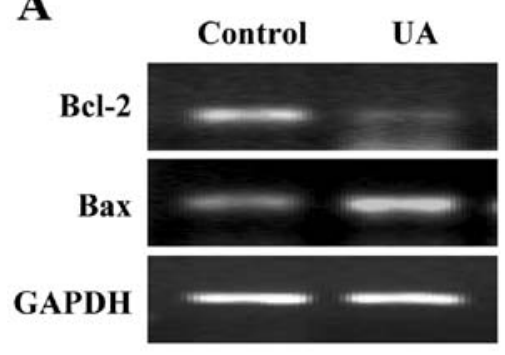

C

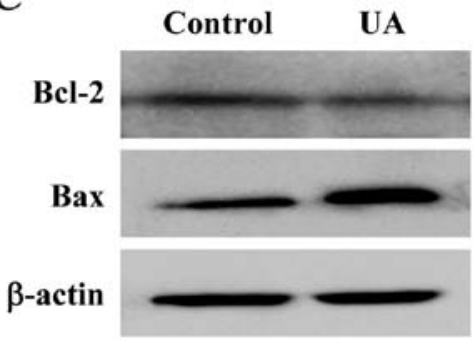

B

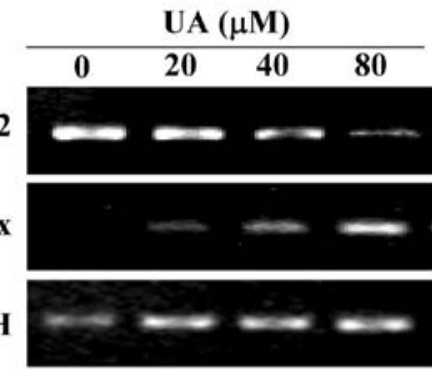

D

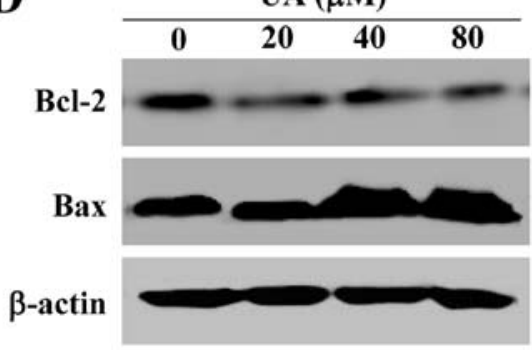

Figure 5. Effect of UA on the expression of Bcl-2 and Bax in CRC xenograft mice and HT-29 cells. The mRNA levels of Bcl-2 and Bax in tumor tissues (A) or HT-29 cells (B) were determined by RT-PCR. The protein expression levels of Bcl-2 and Bax in tumor tissues (C) or HT-29 cells (D) were determined by western blotting. GAPDH and $\beta$-actin were used as the internal controls for the RT-PCR or western blotting, respectively. Images are representative of 3 mice in each group or of three independent cell-based experiments.
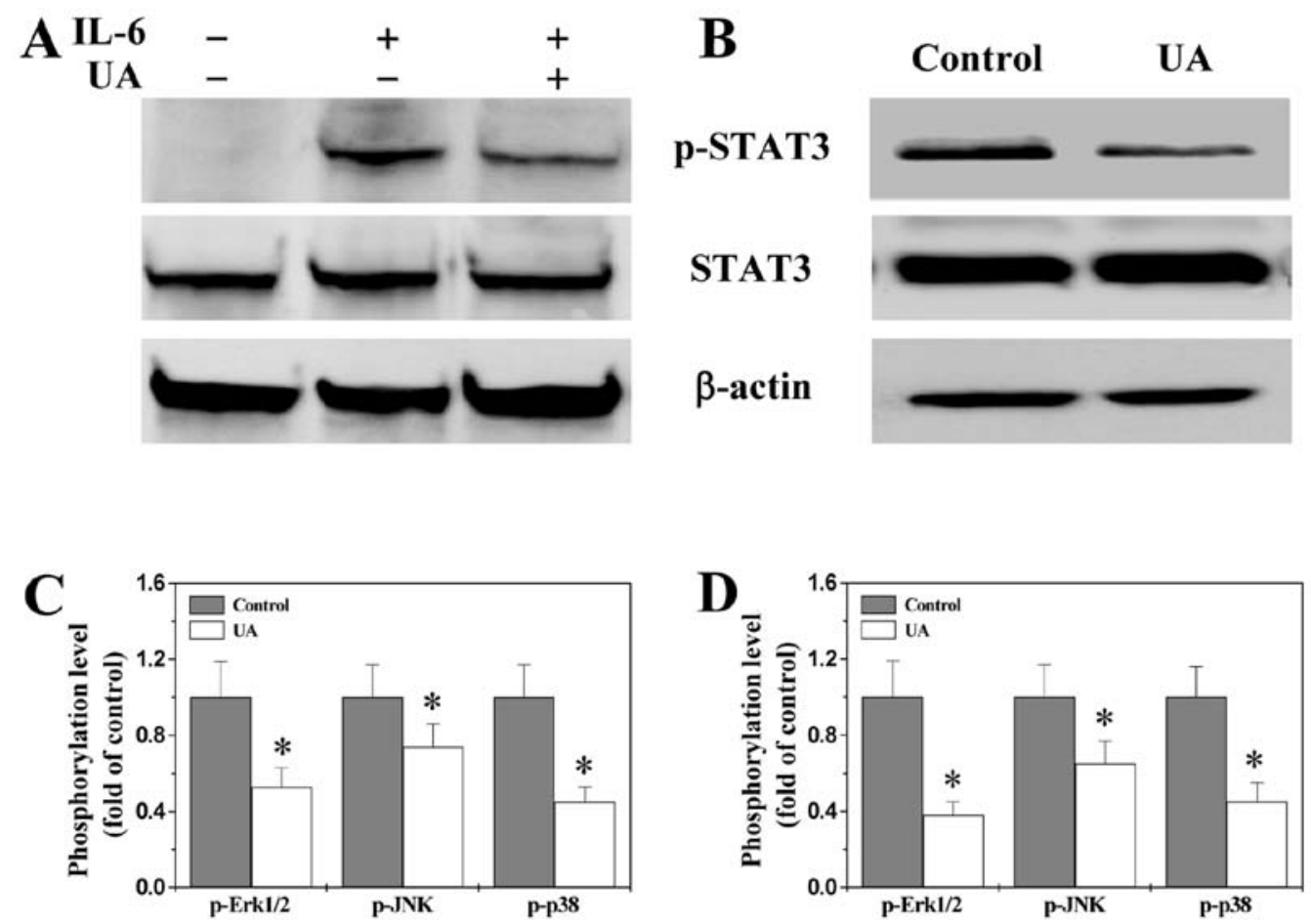

Figure 6. Effect of UA on phosphorylation of STAT3, ERK1/2, JNK and p38 in CRC xenograft mice and HT-29 cells. The level of STAT3 phosphorylation in HT-29 cells (A) and in tumor tissues (B) was determined by western blotting. The phosphorylation levels of ERK1/2, JNK and p38 in tumor tissues (C) and in HT-29 cells (D) were determined by Bio-Plex phosphoprotein assay. The data were normalized to the phosphorylation level within controls and represented as fold of control. Data are averages with SD (error bars) from 8 mice in each group or three independent cell-based experiments. ${ }^{*}<0.01$, versus controls.

\section{Discussion}

Cancer development is strongly correlated with the aberrant activation of multiple intracellular signal transduction pathways which usually function redundantly. In addition, crosstalk between these pathways generates a complicated and robust signaling network that is regulated by compensatory mechanisms. Given the complexity of cancer pathogenesis 
and progression, many of the currently used antitumor agents, which typically target a single intracellular pathway, might not always be effective on complex tumor systems. In contrast, long-term use of these single-target-based agents often generates drug resistance. Moreover, most currently used chemotherapies contain intrinsic toxicity against normal cells. Therefore, the development of novel anticancer chemotherapies is urgently needed. Natural products have received great interest since they have relatively fewer side effects as compared to modern chemotherapeutics and have been shown to display multiple therapeutic effects for various diseases including cancer. Ursolic acid (UA), a major active compound of many traditional Chinese medicinal herbs, has been shown to possess anticancer activity. However, the precise mechanism of its potential tumoricidal activity remains largely unclear. Therefore, before UA can be further developed as an anticancer agent, the mode of action for its antitumor effects should be fully elucidated.

Cancer cells are characterized by an uncontrolled increase in cell proliferation and/or a reduction in cell apoptosis. The mitochondrion-dependent pathway is the most common apoptotic pathway in vertebrate animal cells, which is highly regulated by Bcl-2 family proteins including anti-apoptotic members such as Bcl-2 and pro-apoptotic members such as Bax (27). The ratio of active anti- and pro-apoptotic Bcl-2 family members determines the fate of cells and alteration of the ratio by aberrant expression of these proteins impairs the normal apoptotic program contributing to various apoptosisrelated diseases including cancer $(27,28)$. Higher Bcl-2-to-Bax ratios due to the overexpression of $\mathrm{Bcl}-2$ or downregulation of Bax expression are commonly found in cancer, which not only confers a survival advantage to the cancer cells but also causes resistance to chemo- and radio-therapies. Eukaryotic cell proliferation is primarily regulated by the cell cycle. G1/S transition is one of the two main checkpoints of the cell cycle and is responsible for initiation and completion of DNA replication (29). G1/S progression is strongly regulated by Cyclin D1 that exerts its function via forming an active complex with its CDK major catalytic partners (CDK4/6) (30). An unchecked or hyperactivated Cyclin D1/CDK4 complex often leads to uncontrolled cell division and malignancy $(31,32)$. As a proliferation inhibitor, p21 protein plays a role in $\mathrm{G} 1$ arrest by binding to and inhibiting the activity of Cyclin-CDK complexes; and the decrease of p21 is associated with the promotion of tumor formation and a poor prognosis in many types of cancer (33). Therefore, re-balancing of cell apoptosis and proliferation via regulation of the expression of apoptosis- or cell cycle-related genes is a promising target for cancer chemotherapies. Using a CRC mouse xenograft model and a human colon carcinoma cell line, we demonstrated that UA inhibits cancer growth both in vivo and in vitro, without apparent toxicity. Additionally, UA suppresses cancer cell proliferation through blocking G1/S arrest and promotes cell apoptosis both in tumors of CRC mice and in colon cancer cell line. The pro-apoptotic and anti-proliferative activities of UA were mediated by its effects on the expression of relevant genes. UA treatment profoundly increased the pro-apoptotic $\mathrm{Bax} / \mathrm{Bcl}-2$ ratio, the expression of anti-proliferative $\mathrm{p} 21$ and downregulated the expression of pro-proliferative Cyclin D1 and CDK4.
The processes of apoptosis and cellular proliferation are regulated by multiple intracellular signals including STAT3, ERK, JNK and p38 pathways. Aberrant activation of these pathways is involved in cancer progression. In the present study we demonstrated that UA significantly suppress the activation of these CRC-related signaling pathways both in vivo and in vitro.

In conclusion, we report for the first time that UA possesses a broad range of anticancer activities due to its ability to affect multiple intracellular targets. Our findings suggest that UA could be a novel therapeutic agent for the treatment of colorectal and possibly other cancers.

\section{Acknowledgements}

This study was sponsored by the National Natural Science Foundation of China (no. 81073097), the Developmental Fund of Chen Keji Integrative Medicine (no. CKJ 2011001), and the China Postdoctoral Science Foundation (no. 2012M511437).

\section{References}

1. Jemal A, Bray F, Center MM, Ferlay J, Ward E and Forman D: Global cancer statistics. CA Cancer J Clin 61: 69-90, 2011.

2. Gustin DM and Brenner DE: Chemoprevention of colon cancer: current status and future prospects. Cancer Metastasis Rev 21: 323-348, 2002.

3. Gorlick R and Bertino JR: Drug resistance in colon cancer. Semin Oncol 26: 606-611, 1999.

4. Longley DB, Allen WL and Johnston PG: Drug resistance, predictive markers and pharmacogenomics in colorectal cancer. Biochim Biophys Acta 1766: 184-196, 2006.

5. Aggarwal BB, Kunnumakkara AB, Harikumar KB, Gupta SR, Tharakan ST, Koca C, Dey S and Sung B: Signal transducer and activator of transcription-3, inflammation, and cancer: how intimate is the relationship? Ann NY Acad Sci 1171: 59-76, 2009.

6. Zhong Z, Wen Z and Darnell J: Stat3: a STAT family member activated by tyrosine phosphorylation in response to epidermal growth factor and interleukin-6. Science 264: 95-98, 1994.

7. Bromberg J and Wang TC: Inflammation and cancer: IL- 6 and STAT3 complete the link. Cancer Cell 15: 79-80, 2009.

8. Kusaba T, Nakayama T, Yamazumi K, Yakata Y, Yoshizaki A, Inoue K, Nagayasu T and Sekine I: Activation of STAT3 is a marker of poor prognosis in human colorectal cancer. Oncol Rep 15: 1445-1451, 2006.

9. Lin Q, Lai R, Chirieac LR, Li C, Thomazy VA, Grammatikakis I, Rassidakis GZ, Zhang W, Fujio Y, Kunisada K, Hamilton SR and Amin HM: Constitutive activation of JAK3/STAT3 in colon carcinoma tumors and cell lines: inhibition of JAK3/ STAT3 signaling induces apoptosis and cell cycle arrest of colon carcinoma cells. Am J Pathol 167: 969-980, 2005.

10. Xiong H, Zhang Z, Tian X, Sun D, Liang Q, Zhang Y, Lu R, Chen $Y$ and Fang J: Inhibition of JAK1, 2/STAT3 signaling induces apoptosis, cell cycle arrest, and reduces tumor cell invasion in colorectal cancer cells. Neoplasia 10: 287-297, 2008.

11. Sebolt-Leopold JS: Development of anticancer drugs targeting the MAP kinase pathway. Oncogene 19: 6594-6599, 2000.

12. Seger R and Krebs EG: The MAPK signaling cascade. FASEB J 9: 726-735, 1995.

13. Taupin D and Podolski DK: Mitogen-activated protein kinase activation regulates intestinal epithelial differentiation. Gastroenterology 116: 1072-1080, 1999.

14. Wang X, Wang Q, Hu W and Evers BM: Regulation of phorbol estermediated TRAF1 induction in human colon cancer cells through a PKC/RAF/ERK/NF-kappaB-dependent pathway. Oncogene 23: 1885-1895, 2004.

15. Roberts PJ and Der CJ: Targeting the Raf-MEK-ERK mitogenactivated protein kinase cascade for the treatment of cancer. Oncogene 26: 3291-3310, 2007.

16. Schwartsmann G, Di Leone LP, Dal Pizzol F and Roesler R: MAPK pathway activation in colorectal cancer: a therapeutic opportunity for GRP receptor antagonists. Lancet Oncol 6: 444-445, 2005. 
17. Fang JY and Richardson BC: The MAPK signalling pathways and colorectal cancer. Lancet Oncol 6: 322-327, 2005.

18. Ma XH and Wang ZW: Anticancer drug discovery in the future: an evolutionary perspective. Drug Discov Today 14: 1136-1142, 2009.

19. Gordaliza M: Natural products as leads to anticancer drugs. Clin Transl Oncol 9: 767-776, 2007.

20. Lin JM, Chen YQ, Wei LH, Chen XZ, Xu W, Hong ZF, Sferra TJ and Peng J: Hedyotis Diffusa Willd extract induces apoptosis via activation of the mitochondrion-dependent pathway in human colon carcinoma cells. Int J Oncol 37: 1331-1338, 2010.

21. Cai QY, Lin JM, Wei LH, Zhang L, Wang LL, Zhan YZ, Zeng JW, $\mathrm{Xu}$ W, Shen AL, Hong ZF and Peng J: Hedyotis diffusa Willd inhibits colorectal cancer growth in vivo via inhibition of STAT3 signaling pathway. Int J Mol Sci 13: 6117-6128, 2012.

22. Wei LH, Chen YQ, Lin JM, Zhao JY, Chen XZ, Xu W, Liu XX, Sferra TJ and Peng J: Scutellaria Barbata D. Don induces apoptosis of human colon carcinoma cell via activation of the mitochondrion-dependent pathway. J Med Plants Res 5: 1962-1970, 2011.

23. Zheng LP, Chen YQ, Lin W, Zhuang QC, Chen XZ, Xu W, Liu XX, Peng J and Sferra TJ: Spica Prunellae extract promotes mitochondrion-dependent apoptosis in a human colon carcinoma cell line. Afr J Pharm Pharmacol 5: 327-335, 2011.

24. Ikeda Y, Murakami A and Ohigashi H: Ursolic acid: an anti- and pro-inflammatory triterpenoid. Mol Nutr Food Res 52: 26-42, 2008

25. Xavier CP, Lima CF, Preto A, Seruca R, Fernandes-Ferreira M and Pereira-Wilson C: Luteolin, quercetin and ursolic acid are potent inhibitors of proliferation and inducers of apoptosis in both KRAS and BRAF mutated human colorectal cancer cells. Cancer Lett 281: 162-170, 2009.
26. Andersson D, Liu JJ, Nilsson A and Duan RD: Ursolic acid inhibits proliferation and stimulates apoptosis in HT29 cells following activation of alkaline sphingomyelinase. Anticancer Res 23: 3317-3322, 2003.

27. Adams J and Cory S: The Bcl-2 apoptotic switch in cancer development and therapy. Oncogene 26: 1324-1337, 2007.

28. Youle RJ and Strasser A: The BCL-2 protein family: opposing activities that mediate cell death. Nat Rev Mol Cell Biol 9: 47-59, 2008.

29. Nurse P: Ordering $S$ phase and $M$ phase in the cell cycle. Cell 79: 547-550, 1994.

30. Morgan DO: Principles of CDK regulation. Nature 374: 131-134, 1995.

31. Zafonte BT, Hulit J, Amanatullah DF, Albanese C, Wang C, Rosen E, Reutens A, Sparano JA, Lisanti MP and Pestell RG: Cell-cycle dysregulation in breast cancer: breast cancer therapies targeting the cell cycle. Front Biosci 5: D938-D961, 2000.

32. Kouraklis G, Theocharis S, Vamvakas P, Vagianos C, Glinavou A Giaginis C and Sioka C: Cyclin D1 and Rb protein expression and their correlation with prognosis in patients with colon cancer. World J Surg Oncol 4: 5, 2006.

33. Domagala W, Welcker M, Chosia M, Karbowniczek M, Harezga B, Bartkova J, Bartek J and Osborn M: p21/WAF1/Cip1 expression in invasive ductal breast carcinoma: Relationship to p53, proliferation rate, and survival at 5 years. Virchows Arch 439: 132-140, 2001. 